\title{
A remark on conditioned invariance in the behavioral approach*
}

\author{
Ricardo Pereira ${ }^{1}$ and Paula Rocha ${ }^{2}$
}

\begin{abstract}
In this paper a definition for the property of behavioral invariance is proposed with the purpose of generalizing the state space geometric approach to the behavioral setting. Based on this notion together with the well-known notion of behavioral observer, a definition of conditioned invariance is also presented. The results obtained for the characterization of the defined properties put into evidence some problems that, in our opinion, should deserve attention. This could serve as a starting point for a discussion on the foundations of an analogue of the geometric theory within the behavioral setting.
\end{abstract}

\section{INTRODUCTION}

In this paper we make an analysis and raise some questions on the extension of the fundamental notions of invariance and conditioned invariance from the geometric theory (see [1]) to the case of behavioral systems.

Basically, given two behaviors $\mathcal{B}$ and $\mathcal{V}$ we shall say that $\mathcal{V}$ is $\mathcal{B}$-invariant if the dynamics of $\mathcal{B}$ is autonomous modulo $\mathcal{V}$. This seems to combine well with the definition of behavioral (tracking) observer ([8], [7]) in order to produce a good definition of conditioned invariance. Indeed, according to [8], [7], very roughly speaking, an estimator is an observer if the corresponding error behavior is autonomous. On the other hand, in the state space setting a subspace $\mathcal{V}_{\mathcal{X}}$ of the state space $\mathcal{X}$ is said to be conditioned invariant if it is invariant with respect to the error dynamics of some observer. This motivates us to similarly define conditioned invariance in the behavioral context as (behavioral) invariance with respect to the error dynamics of some (behavioral) observer.

The behavioral definitions that we propose are related to the ones in the state space setting precisely in the same way as the behavioral definition of (tracking) observer from [8] and [7] is. Therefore, similarly to what happens with behavioral

\footnotetext{
*Supported in part by FEDER funds through COMPETE - Operational Programme Factors of Competitiveness ("Programa Operacional Factores de Competitividade") and by Portuguese funds through the Center for Research and Development in Mathematics and Applications and the Portuguese Foundation for Science and Technology "FCT-Fundação para a Ciência e Tecnologia"), within project PEst-C/MAT/UI4106/2011 with COMPETE number FCOMP-01-0124-FEDER-022690.

${ }^{1} \mathrm{R}$. Pereira is with the Center for Research and Development in Mathematics and Applications, Department of Mathematics, University of Aveiro, Portugal ricardopereira@ua.pt

${ }^{2} \mathrm{P}$. Rocha is with the Center for Research and Development in Mathematics and Applications and the Department of Electrical and Computer Engineering, Faculty of Engineering, University of Oporto, Portugal mprochadfe.up.pt
}

observers, when particularized to state space systems, the behavioral properties of invariance and conditioned invariance do not coincide with the classical ones. This by itself should not constitute a problem, since the same happens for some other "well established" behavioral properties.

However, speaking in loose terms, it turns out that: $(i)$ the autonomy of a behavior $\mathcal{B}$ is sufficient for the $\mathcal{B}$-invariance of any behavior $\mathcal{V}$; (ii) the existence of an observer guarantees the conditioned invariance of any behavior in the universe of the to-be-estimated variable.

This seems to indicate that the behavioral properties of invariance and conditioned invariance are not strong enough.

On the other hand, given the coherence of these properties with the definition of behavioral (tracking) observer it does not appear to be easy to find good alternatives.

Rather than presenting answers, the aim of this paper is to set a starting point for a discussion on these issues within the research community.

The exposition is organized as follows: Section II introduces the relevant preliminaries on behaviors; the behavioral notion of invariance is introduced in Section III; Section IV gives an overview of behavioral (tracking) observers; Section V is dedicated to conditioned invariance; finally, Section VI contains our concluding remarks.

\section{PRELIMINARIES}

In the behavioral approach [9] a dynamical system is defined as a quadruple $\Sigma=(\mathcal{T}, \mathcal{W}, \mathcal{U}, \mathcal{B})$, where $\mathcal{T}$ is the time axis, $\mathcal{W}$ is the signal space, and the behavior $\mathcal{B}$ is a subset of a universe $\mathcal{U} \subset \mathcal{W}^{\mathcal{T}}=\{f: \mathcal{T} \rightarrow \mathcal{W}\}$. The elements of $\mathcal{B}$ are called trajectories.

In this paper we will only consider linear time-invariant differential systems and the term behavior will always refer to the behavior of such systems. A linear time-invariant differential system is a dynamical system with time axis $\mathcal{T}=$ $\mathbb{R}$, signal space $\mathcal{W}=\mathbb{R}^{\mathrm{w}}$, for some $\mathrm{w} \in \mathbb{N}$, and the behavior $\mathcal{B}$ is a linear subspace of the universe $\mathcal{U}=\mathcal{C}^{\infty}\left(\mathbb{R}, \mathbb{R}^{w}\right)$ consisting of all solutions of a linear, homogeneous matrix differential equation with constant coefficients. This means that there exists a positive integer $g$ and a polynomial matrix $R(s) \in \mathbb{R}^{g \times \mathrm{w}}[s]$ such that

$$
\mathcal{B}=\left\{w \in \mathcal{C}^{\infty}\left(\mathbb{R}, \mathbb{R}^{w}\right): R(\sigma) w=0\right\},
$$


where $\sigma$ denotes the differential operator $\frac{d}{d t}$.

The polynomial matrix $R(s)$ is called a kernel representation matrix of $\mathcal{B}$ and we write $\mathcal{B}=\operatorname{ker} R(\sigma)$. For short, whenever the context is clear we omit the indeterminate $s$ and the operator $\sigma$. Note that different representations may give rise to the same behavior. In particular $\operatorname{ker} R=\operatorname{ker} U R$ for any unimodular matrix $U$. Moreover, $\mathcal{B}_{1}=\operatorname{ker} R_{1} \subset$ $\mathcal{B}_{2}=\operatorname{ker} R_{2}$ if and only if there exists a polynomial matrix $\bar{R}$ such that $R_{2}=\bar{R} R_{1}$.

A behavior $\mathcal{B}$ is called autonomous if the future of any trajectory in $\mathcal{B}$ is completely determined by its past. The formal definition of autonomy is given next.

Definition 1: A behavior $\mathcal{B}$ is autonomous if for every $w \in \mathcal{B}$ we have that $w(t)=0$ for all $t \leq 0$ implies $w=0$.

It was shown in [5] that if $\mathcal{B}=\operatorname{ker} R$, then $\mathcal{B}$ is autonomous if and only if $R$ has full column rank.

\section{INVARIANCE}

Before introducing the definition of invariance in the behavioral context, we recall the classical state space case. Consider an autonomous state space system $\dot{x}=A x$, with state space $\mathcal{X}$, where $x(t) \in \mathbb{R}^{n}$ is the state vector at time $t$ and $A \in \mathbb{R}^{n \times n}$. This model defines a behavior $\mathcal{B}=\operatorname{ker}(\sigma I-A)$. The notion of invariance is defined as follows [1].

Definition 2: A subspace $\mathcal{V}_{\mathcal{X}}$ of $\mathcal{X}$ is said to be $A$ invariant if

$$
\left[x(0) \in \mathcal{V}_{\mathcal{X}}, x \in \mathcal{B}\right] \Rightarrow\left[x(t) \in \mathcal{V}_{\mathcal{X}}, \forall t \geq 0\right] .
$$

In the behavioral case, instead of considering the invariance of a subspace of the state space under the dynamics induced by state space equations, we consider the invariance of a given behavior $\mathcal{V}$ under the dynamics associated to a behavior $\mathcal{B}$. Basically, the role of the state space $\mathcal{X}$ will now be played by the universe $\mathcal{U}$, and the subspaces $\mathcal{V}_{\mathcal{X}}$ will be replaced by behaviors $\mathcal{V}$.

Definition 3: Given two behaviors $\mathcal{B}$ and $\mathcal{V}$, we say that $\mathcal{V}$ is $\mathcal{B}$-invariant if the following condition holds:

$$
\left[w_{\mid(-\infty, 0]} \in \mathcal{V}_{\mid(-\infty, 0]}, w \in \mathcal{B}\right] \Rightarrow[w \in \mathcal{V}]
$$

where, as usual, $w_{\mid(-\infty, 0]}$ denotes the restriction of $w$ to the interval $(-\infty, 0]$ and $\mathcal{V}_{\mid(-\infty, 0]}$ is the set of restrictions of the trajectories $v \in \mathcal{V}$ to that same interval.

This means that, when $\mathcal{V}$ is $\mathcal{B}$-invariant, a trajectory $w$ which has a past compatible with $\mathcal{V}$ (i.e., $\left.w_{\mid(-\infty, 0]} \in \mathcal{V}_{\mid(-\infty, 0]}\right)$ and, moreover, evolves according to the dynamics of $\mathcal{B}$ (i.e., $w \in \mathcal{B}$ ) must remain in $\mathcal{V}$ in the future.
In order to characterize the invariance of behaviors we recall the notion of hermetic sub-behavior presented in [3] in the context of multidimensional (nD) systems, now adapted to the $1 \mathrm{D}$ case.

Definition 4: Let $\mathcal{B}$ be a behavior. A sub-behavior $\mathcal{B}^{\prime}$ of $\mathcal{B}$ is said to be hermetic if, for every $t_{0} \in \mathbb{R}$ :

$$
\left[w \in \mathcal{B}, w_{\mid\left(-\infty, t_{0}\right]} \in \mathcal{B}_{\mid\left(-\infty, t_{0}\right]}^{\prime}\right] \Rightarrow\left[w \in \mathcal{B}^{\prime}\right] .
$$

Taking time invariance into account, the next result follows immediately from [3, Thm. 7].

Proposition 5: Let $\mathcal{B}$ and $\mathcal{V}$ be two behaviors. The following statements are equivalent:

1) $\mathcal{V}$ is $\mathcal{B}$-invariant.

2) $(\mathcal{B} \cap \mathcal{V})$ is a hermetic sub-behavior of $\mathcal{B}$.

3) $\mathcal{B} /(\mathcal{B} \cap \mathcal{V})$ is autonomous.

Remark 6: It follows from condition 2 in this proposition that if $\mathcal{B}$ is autonomous then any behavior $\mathcal{V}$ is $\mathcal{B}$-invariant.

According to [2, Thm. 2.56], if $\mathcal{B}^{\prime}$ is a sub-behavior of $\mathcal{B}$, then the factor module $\mathcal{B} / \mathcal{B}^{\prime}$ also admits the structure of a behavior. Hence, $\mathcal{B}$-invariance can also be characterized in terms of kernel representations. Indeed, let $\mathcal{B}=\operatorname{ker} R$ and $\mathcal{V}=\operatorname{ker} V$, where $R$ and $V$ are polynomial matrices of suitable dimensions. It is not difficult to show that a polynomial matrix $E$ of full row rank is a kernel representation of the behavior $\mathcal{B} \cap \mathcal{V}$ if and only if there exist polynomial matrices $\bar{R}$ and $\bar{V}$ such that

$$
R=\bar{R} E ; V=\bar{V} E \text { and } \operatorname{ker}\left[\begin{array}{l}
\bar{R} \\
\bar{V}
\end{array}\right]=\{0\} .
$$

Consequently, by [4, Lemma 2.13], the factor module $\mathcal{B} /(\mathcal{B} \cap \mathcal{V})$ is isomorphic to $\operatorname{ker} \bar{R}$. This leads to the following characterization.

Proposition 7: Given two behaviors $\mathcal{B}=\operatorname{ker} R$ and $\mathcal{V}$, let $E$ and $\bar{R}$ be polynomial matrices such that $\mathcal{B} \cap \mathcal{V}=\operatorname{ker} E$ and $R=\bar{R} E$, where $E$ has full row rank. Then the following statements are equivalent:

1) $\mathcal{V}$ is $\mathcal{B}$-invariant.

2) the matrix $\bar{R}$ is full column rank.

The next example shows that behavioral invariance does not coincide with the classical notion of invariance for state space systems.

Example 8: Let $\mathcal{B}$ be given by

$$
\dot{x}=A x
$$

with $A=\left[\begin{array}{ll}0 & 1 \\ 1 & 1\end{array}\right]$, and consider $\mathcal{V}=\operatorname{ker}\left[\begin{array}{ll}0 & 1\end{array}\right]$. Then, $\mathcal{V}$ is clearly not $A$-invariant according to Definition 2 . On the 
other hand, by Definition $3, \mathcal{V}$ is $\mathcal{B}$-invariant when viewed as a subspace of $\mathcal{U}=\mathcal{C}^{\infty}\left(\mathbb{R}, \mathbb{R}^{2}\right)$. This happens because

$$
\left[x_{\mid(-\infty, 0]} \in \mathcal{V}_{\mid(-\infty, 0]}, x \in \mathcal{B}\right] \Rightarrow x \equiv 0 \Rightarrow x \in \mathcal{V}
$$

As it will be seen in the next section, the same kind of difference occurs between observers defined in the classical state space setting and behavioral observers.

\section{OBSERVERS}

In this section we recall some elementary notions of the theory of behavioral observers developed in [7] and [8].

For this purpose it is necessary to consider systems with different variables. Therefore, in the sequel, when denoting a behavior the corresponding system variable will be made explicit by means of a subscript.

Consider a linear time-invariant differential system with behavior $\mathcal{B}_{\left(w_{1}, w_{2}\right)}$, where the system variable $w=\left(w_{1}, w_{2}\right)$ is partitioned into measured variables $w_{1}$ and to-be-estimated variables $w_{2}$. An observer of $w_{2}$ from $w_{1}$ for $\mathcal{B}_{\left(w_{1}, w_{2}\right)}$ is defined as follows.

Definition 9: Given a linear time-invariant differential behavior $\mathcal{B}_{\left(w_{1}, w_{2}\right)}$, let $\widehat{\mathcal{B}}_{\left(w_{1}, \widehat{w}_{2}\right)}$ be a behavior such that the universe $\mathcal{U}_{\widehat{w}_{2}}$ coincides with the universe $\mathcal{U}_{w_{2}}$ of the variable $w_{2} . \widehat{\mathcal{B}}_{\left(w_{1}, \widehat{w}_{2}\right)}$ is said to be an observer of $w_{2}$ from $w_{1}$ for $\mathcal{B}_{\left(w_{1}, w_{2}\right)}$ if whenever $\left(w_{1}, w_{2}\right) \in \mathcal{B}_{\left(w_{1}, w_{2}\right)}$ and $\left(w_{1}, \widehat{w}_{2}\right) \in$ $\widehat{\mathcal{B}}_{\left(w_{1}, \widehat{w}_{2}\right)}$ with $\widehat{w}_{2}(t)=w_{2}(t)$ for $t \in(-\infty, 0]$, then $\widehat{w}_{2}(t)=$ $w_{2}(t), \forall t \in \mathbb{R}$.

Remark 10: This corresponds to the definition of tracking observer given in [7]. Defining the error behavior as

$$
\begin{aligned}
\mathcal{B}_{e}= & \left\{e=\widehat{w}_{2}-w_{2}: \exists w_{1}\right. \text { s.t. } \\
& \left.\left(w_{1}, w_{2}\right) \in \mathcal{B}_{\left(w_{1}, w_{2}\right)},\left(w_{1}, \widehat{w}_{2}\right) \in \widehat{\mathcal{B}}_{\left(w_{1}, \widehat{w}_{2}\right)}\right\},
\end{aligned}
$$

it is clear that $\widehat{\mathcal{B}}_{\left(w_{1}, \widehat{w}_{2}\right)}$ is an observer of $w_{2}$ from $w_{1}$ for $\mathcal{B}_{\left(w_{1}, w_{2}\right)}$ if and only if $\mathcal{B}_{e}$ is autonomous, i.e., if and only if

$$
\left[e \in \mathcal{B}_{e}, e_{\mid(-\infty, 0]} \equiv 0\right] \Rightarrow[e(t)=0, \forall t \in \mathbb{R}] \text {. }
$$

In case an observer of $w_{2}$ from $w_{1}$ for $\mathcal{B}_{\left(w_{1}, w_{2}\right)}$ exists, we shall say that $w_{2}$ is trackable from $w_{1}$ in $\mathcal{B}_{\left(w_{1}, w_{2}\right)}$. In [8], the following test is given for trackability.

Proposition 11: Let $\mathcal{B}_{\left(w_{1}, w_{2}\right)}$ be described by

$$
R_{2}(\sigma) w_{2}=R_{1}(\sigma) w_{1}
$$

with $R_{1} \in \mathbb{R}^{g \times \mathrm{w}_{1}}[s]$ and $R_{2} \in \mathbb{R}^{g \times \mathrm{w}_{2}}[s]$ polynomial matrices, then there exists an observer of $w_{2}$ from $w_{1}$ for $\mathcal{B}_{\left(w_{1}, w_{2}\right)}$ if and only if $R_{2}$ has full column rank.

Remark 12: Note that $\operatorname{ker} R_{2}$ coincides with the hidden behavior of $w_{2}$ defined in [7],

$$
\mathcal{N}_{w_{2}}\left(\mathcal{B}_{\left(w_{1}, w_{2}\right)}\right)=\left\{w_{2} \mid\left(0, w_{2}\right) \in \mathcal{B}_{\left(w_{1}, w_{2}\right)}\right\} .
$$

Hence the proposition means that trackability is equivalent to the autonomy of the hidden behavior, cf [7, Def. 4.2 and Prop. 4.3].

In order to make a comparison with the notion of observer for state space systems, we next recall the definition of state observer.

Definition 13: Consider a state space system $\Sigma$ with behavior $\mathcal{B}_{(y, x)}$ described by

$$
\left\{\begin{array}{l}
\dot{x}=A x \\
y=C x
\end{array}\right.
$$

with state space $\mathcal{X}$, where $x$ is the state (to be estimated), $y$ is the (measured) output, and $A$ and $C$ are real matrices of suitable dimensions.

A system $\Omega$ with state space $\widehat{\mathcal{X}}=\mathcal{X}$, and behavior $\widehat{\mathcal{B}}_{(y, \widehat{x})}$ given by the equation

$$
\dot{\widehat{x}}=P \widehat{x}+R y
$$

is said to be a state observer for $\Sigma$ if, defining the error of the estimate by $e=\widehat{x}-x$, for any pair of initial values $(x(0), \widehat{x}(0))$ satisfying $e(0)=0$, we have $e(t)=0$ for all $t \geq 0$.

This is a simplified version of the definitions presented in the literature (as, for instance, the one given in [6], where the dynamics of the state estimate $\widehat{x}$ is not necessarily of first order).

Remark 14: Defining the error behavior $\mathcal{B}_{e}^{s s}=\{e=\widehat{x}-$ $x: \exists y$ s.t. $\left.(y, x) \in \mathcal{B}_{(y, x)},(y, \widehat{x}) \in \widehat{\mathcal{B}}_{(y, \widehat{x})}\right\}$, saying that $\Omega$ is a state observer for $\Sigma$ means that the following condition is satisfied:

$$
\left[e(0)=0, e \in \mathcal{B}_{e}^{s s}\right] \Rightarrow[e(t)=0, \forall t \in \mathbb{R}]
$$

As already mentioned, when applied to state space systems, the behavioral notion of observer does not coincide with the one of state observer (cf Definition 13). This can be illustrated by the following simple example.

Example 15: Let $\mathcal{B}_{(y, x)}$ be described by

$$
\left\{\begin{array}{l}
\dot{x}=x \\
y=x
\end{array}\right.
$$

and $\widehat{\mathcal{B}}_{(y, \widehat{x})}$ by

$$
\dot{\widehat{x}}=0 \widehat{x}+0 y
$$

This behavior is an observer for $x$ from $y$ for $\mathcal{B}_{(y, x)}$ in the behavioral setting, but it is not a state observer for $\mathcal{B}_{(y, x)}$. Indeed, note that the $x$ trajectories in $B_{(y, x)}$ are of the form $x(t)=e^{t} x(0)$, whereas the $\widehat{x}$ trajectories in $\widehat{\mathcal{B}}_{(y, \widehat{x})}$ are constant. Therefore

$$
\widehat{x}(t)=x(t) \text { for } t \in(-\infty, 0] \Rightarrow \widehat{x} \equiv x \equiv 0 .
$$


However, for the trajectories $x(t)=e^{t}$ and $\widehat{x}(t) \equiv 1$ it happens that

$$
[x(0)=\widehat{x}(0)], \text { but }\left[x(t)=e^{t} \neq 1=\widehat{x}(t) \text { for } t>0\right] .
$$

\section{CONDITIONED INVARIANCE}

The aim of this section is to introduce conditioned invariance in the behavioral framework based on the definitions of behavioral invariance (cf Definition 3) and behavioral observer (cf Definition 9), and of the notion of conditioned invariance defined in the context of state space systems ([1],[6]). This latter rests on the concept of observer modulo a subspace of the state space defined next.

Definition 16: Consider a state space system $\Sigma$ described by (1). A system $\Omega$ with equation (2) is said to be an observer for $\mathcal{X} / \mathcal{V}_{\mathcal{X}}$, where $\mathcal{V}_{\mathcal{X}}$ is a subspace of $\mathcal{X}$, if, for any pair of initial values $\left(x_{0}, \widehat{x}_{0}\right)$ satisfying $\widehat{x}_{0}-x_{0} \in \mathcal{V}_{\mathcal{X}}$, we have $\widehat{x}(t)-x(t) \in \mathcal{V}_{\mathcal{X}}$ for all $t \geq 0$.

In terms of the previously defined error behavior $\mathcal{B}_{e}^{s s}$, the condition in this definition can be restated as

$$
\left[e(0) \in \mathcal{V}_{\mathcal{X}}, e \in \mathcal{B}_{e}^{s s}\right] \Rightarrow\left[e(t) \in \mathcal{V}_{\mathcal{X}}, \forall t \in \mathbb{R}\right]
$$

which corresponds to the invariance of $\mathcal{V}_{\mathcal{X}}$ under the error dynamics as defined in the state space context, see Definition 2.

Note that, if $\mathcal{V}_{\mathcal{X}}$ is a subspace of $\mathcal{X}$, an observer for $\mathcal{X} / \mathcal{V}_{\mathcal{X}}$ may not exist.

Definition 17: A subspace $\mathcal{V}_{\mathcal{X}}$ of $\mathcal{X}$ is called conditioned invariant if there exists an observer for $\mathcal{X} / \mathcal{V}_{\mathcal{X}}$

Given the behavioral definition of observer (Definition 9) and the classical definition of observer for $\mathcal{X} / \mathcal{V}_{\mathcal{X}}$ (Definition 16) the following behavioral definition of observer modulo $\mathcal{V}$ arises naturally.

Definition 18: Let $\mathcal{B}_{\left(w_{1}, w_{2}\right)}$ and $\widehat{\mathcal{B}}_{\left(w_{1}, \widehat{w}_{2}\right)}$ be two linear time-invariant differential behaviors for which the universes $\mathcal{U}_{w_{2}}$ and $\mathcal{U}_{\widehat{w}_{2}}$ of the variables $w_{2}$ and $\widehat{w}_{2}$, resp., coincide. Define the error behavior $\mathcal{B}_{e}$ as in Remark 10, and let $\mathcal{V}_{e}$ be a behavior with variable $e$. The behavior $\widehat{\mathcal{B}}_{\left(w_{1}, \widehat{w}_{2}\right)}$ is said to be an observer of $w_{2}$ from $w_{1}$ for $\mathcal{B}_{\left(w_{1}, w_{2}\right)}$ modulo $\mathcal{V}_{e}$ if

$$
\left[\begin{array}{l}
e_{\mid(-\infty, 0]} \in\left(\mathcal{V}_{e}\right)_{\mid(-\infty, 0]} \\
e \in \mathcal{B}_{e}
\end{array}\right] \Rightarrow\left[e \in \mathcal{V}_{e}\right]
$$

Remark 19: The previous definition of observer modulo $\mathcal{V}_{e}$ corresponds to saying that $\mathcal{V}_{e}$ is $\mathcal{B}_{e}$-invariant.

Analogously to the state space case, in the behavioral context we define the conditioned invariance of a behavior
$\mathcal{V}$ as the existence of a behavioral observer modulo $\mathcal{V}$.

Definition 20: Let $\mathcal{B}_{\left(w_{1}, w_{2}\right)}$ be a linear time-invariant differential behavior with measured variable $w_{1}$, and tobe-estimated variable $w_{2}$ in a universe $\mathcal{U}_{w_{2}}$. A behavior $\mathcal{V} \subset \mathcal{U}_{w_{2}}$ is said to be conditioned invariant if there exists a (behavioral) observer of $w_{2}$ from $w_{1}$ for $\mathcal{B}_{\left(w_{1}, w_{2}\right)}$ modulo $\mathcal{V}$.

It follows from the previous definitions that, given $\mathcal{B}_{\left(w_{1}, w_{2}\right)}$, a behavior $\mathcal{V} \subset \mathcal{U}_{w_{2}}$ is conditioned invariant with respect to $\mathcal{B}_{\left(w_{1}, w_{2}\right)}$, if there is an observer of $w_{2}$ from $w_{1}$ for $\mathcal{B}_{\left(w_{1}, w_{2}\right)}$ such that $\mathcal{V}$ is invariant with respect to the corresponding error behavior $\mathcal{B}_{e}$. Therefore, in order to characterize conditioned invariance it is important to know which error behaviors can be obtained by designing a suitable observer. This question has been addressed in [7].

Definition 21: Let $\mathcal{B}_{\left(w_{1}, w_{2}\right)}$ be a linear time-invariant differential behavior with observed variable $w_{1}$, and to-beestimated variable $w_{2}$ in a universe $\mathcal{U}_{w_{2}}$. A behavior $\mathcal{E} \subset \mathcal{U}_{w_{2}}$ is said to be an achievable error behavior if there exists an observer $\widehat{\mathcal{B}}_{\left(w_{1}, \widehat{w}_{2}\right)}$ of $w_{1}$ from $w_{2}$ with error behavior $\mathcal{B}_{e}$ such that $\mathcal{E}=\mathcal{B}_{e}$.

Proposition 22: [7, Prop. 3.5] Let $\mathcal{B}_{\left(w_{1}, w_{2}\right)}$ be a linear time-invariant differential behavior with observed variable $w_{1}$, and to-be-estimated variable $w_{2}$ in a universe $\mathcal{U}_{w_{2}}$. Then the behavior $\mathcal{E} \subset \mathcal{U}_{w_{2}}$ is an achievable error behavior if and only if $\mathcal{N}_{w_{2}}\left(\mathcal{B}_{\left(w_{1}, w_{2}\right)}\right) \subset \mathcal{E}$.

Thus $\mathcal{V}$ is conditioned invariant if and only if it is $\mathcal{E}$-invariant, for some achievable error behavior $\mathcal{E}$. By Proposition 22 this immediately leads to the following result.

Proposition 23: Let $\mathcal{B}_{\left(w_{1}, w_{2}\right)}$ be behavior with observed variable $w_{1}$, and to-be-estimated variable $w_{2}$ in a universe $\mathcal{U}_{w_{2}}$. A behavior $\mathcal{V} \subset \mathcal{U}_{w_{2}}$ is conditioned invariant if and only if there exists a behavior $\mathcal{E} \supset \mathcal{N}_{w_{2}}\left(\mathcal{B}_{\left(w_{1}, w_{2}\right)}\right)$ such that $\mathcal{V}$ is $\mathcal{E}$-invariant.

Now, recall that the existence of an observer is equivalent to the autonomy of the hidden behavior, cf Remark 12. Moreover, by Remark 6 , if the hidden behavior $\mathcal{N}_{w_{2}}\left(\mathcal{B}_{\left(w_{1}, w_{2}\right)}\right)$ is autonomous then any behavior $\mathcal{V} \subset \mathcal{U}_{w_{2}}$ is $\mathcal{N}_{w_{2}}\left(\mathcal{B}_{\left(w_{1}, w_{2}\right)}\right)$-invariant. Since $\mathcal{E}=\mathcal{N}_{w_{2}}\left(\mathcal{B}_{\left(w_{1}, w_{2}\right)}\right)$ is trivially an achievable error behavior, this leads to the following result.

Proposition 24: Let $\mathcal{B}_{\left(w_{1}, w_{2}\right)}$ be a behavior such that an observer for $w_{2}$ from $w_{1}$ exists. Then every behavior $\mathcal{V} \subset$ $\mathcal{U}_{w_{2}}$ is conditioned invariant with respect to $\mathcal{B}_{\left(w_{1}, w_{2}\right)}$.

This result is not similar to what happens in the state space case, where a tracking observer always exists, but 
not every subspace of the state space in conditioned invariant.

\section{CONCLUSIONS}

In this paper we proposed definitions for the properties of invariance and conditioned invariance in a behavioral setting. These definitions fit well with the property of behavioral (tracking) observer previously introduced in [8] and [7]. In fact they seem to be the obvious ones, following logics of passing from the state space to the behavioral setting reflected in [8]; however they do not seem to be powerful enough.

Although not mentioned in this paper, given our definition of behavioral invariance, it is also possible to define a property of controlled invariance for which the same kind of issues arise.

Clearly, the definition of behavioral invariance is what plays a central role in this problematic. The definition proposed here should be viewed as a first attempt, that we hope can serve as a basis for further discussion.

\section{REFERENCES}

[1] G. Basile and G. Marro. Controlled and conditioned invariant subspaces in linear system theory, Journal of Optimization Theory and Applications, 3(5):306-315, 1969.

[2] U. Oberst. Multidimensional constant linear systems, Acta Appl. Math., 20:1-175, 1990.

[3] P. Rocha and J. Wood. A new perspective on controllability properties for dynamical systems, Appl. Math. and Comp. Sci., 7(4):869-879, 1997.

[4] P. Rocha and J. Wood. Trajectory control and interconnection of 1D and nD systems, SIAM J. Control Optim., 40(1):107-134, 2001.

[5] J.W. Polderman and J.C. Willems. Introduction to Mathematical Systems Theory, A behavioral approach, volume 26 of Texts in Applied Mathematics. Springer-Verlag, New York, 1998.

[6] H.L. Trentelman, A.A. Stoorvogel and M.L.J. Hautus. Control Theory for Linear Systems Springer, London, 2001.

[7] J. Trumpf, H.L. Trentelman and J.C. Willems. An internal model principle for observers, Proceedings of the 50th IEEE Conference on Decision and Control and European Control Conference, 3992-3999, 2011.

[8] M.E. Valcher and J. C. Willems. Observer Synthesis in the Behavioral Approach, IEEE Transactions on Automatic Control, 44(12):22972307, 1999.

[9] J. C. Willems. Paradigms and puzzles in the theory of dynamical systems, IEEE Trans. Automat. Control, 36(3):259-294, 1991. 